\title{
Digital DICOM in Dentistry
}

\author{
Jeff Burgess*
}

(Retired) Clinical Assistant Professor, Department of Oral Medicine, University of Washington School of Dental Medicine; (Retired) Attending in Pain Center, University of Washington Medical Center; (Retired) Private Practice in Hawaii and Washington; Director, Oral Care Research Associates, USA

\begin{abstract}
Similar to Medicine, digital communication, information processing, and $\mathrm{x}$-ray imaging have changed the face of dentistry. The incorporation of digital systems into medical and dental practice has necessitated development of a standard that allows reliable transmission of information between the devices taking the images, devices storing the images, and devices displaying the images. This standard is termed as DICOM. The following article briefly reviews how DICOM came about, how dentistry is involved, the various elements that are part of the DICOM system, and how DICOM is currently used in dentistry.
\end{abstract}

Keywords: DICOM, digital radiography, digital imaging.

\section{INTRODUCTION}

Similar to medicine, digital x-ray surveys are now quite commonplace in the assessment of dental caries and oral pathology as well as pre-surgical and pre-orthodontic treatment assessment [1, 2] (Fig. 1). With legacy (non-digital) imaging the information that is included with a single film or imaging study is limited in nature, often including only the patient's name and the date of the study. In contrast, digital imaging allows for inclusion of considerable information. This can include not only the above data but also pretty much any other type of information that is desired. Legacy imaging includes the $\mathrm{x}$-ray machine which takes the image and the developer which produces a film. However, with digital systems there is reliance on many different components including the machines that take the images, devices that display the images and devices that store the images. Getting them all to work together so that the resulting 'picture' can be properly displayed that can be problematic without standardization.

So to provide consistency, the American College of Radiology (ACR) and National Electrical Manufacturers Association (NEMA) developed standards to be incorporated into the transmission of digital images. Their efforts ultimately defined what is termed the 'DICOM' standard (Digital Imaging and Communication in Medicine) which has become the worldwide norm for digital image acquisition, storage, and display in Medicine [3].

With the increasingly widespread 'digitization' of dentistry in terms of image acquisition, the development of sophisticated systems such as 3-D CBCT, optical surface scanning and CAD/CAM systems, and the electronic charting of patient records (EDRs - electronic digital records) the

*Address correspondence to this author at the 2006NE $63^{\text {rd }}$ Street, Seattle Washington 98115, USA; Tel: 206450 2640;

E-mails: oral.care.research.assoc@gmail.com,

jeffreyaburgess@hotmail.com
American and other world Dental Associations have also considered adopting the DICOM standard for use in Dentistry [4].

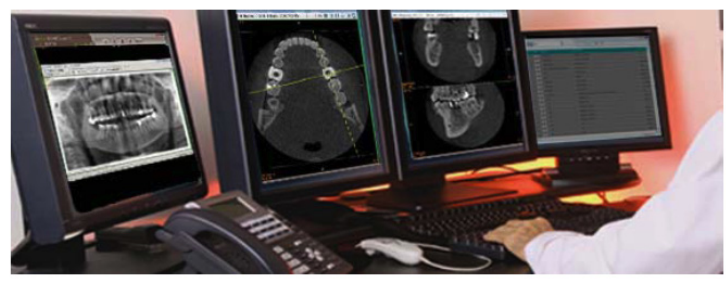

Fig. (1). A DICOM work station showing monitors with different imaging studies and file (courtesy of EBM Technologies).

\section{WHAT IS DICOM}

As noted, DICOM (Digital Imaging and Communication in Medicine) is the standard within Medicine for the transmission of radiologic images and other medical information between computers and various devices that acquire images and also between various equipment and software systems that are produced by different manufacturers [5].

A DICOM image file contains the $\mathrm{x}$-ray image or series of images (for example a multiple slice CBCT imaging study) and other patient related information that is selected from a 'library' of standardized terms (e.g. patient name, identification number, and acquisition modality to name a few) that can be pre-selected. The DICOM library is extensive and continually updated to reflect changing identification standards. A DICOM compliant image file can be thought of as similar to the 'layered' file that is created in the Adobe Photoshop software or a JPEG with the information embedded in the data 'set' or metafile.

\section{History}

It is instructive to briefly review the history of the development of digital standards. Since 1985, when the first itera- 
tion of DICOM was presented for use (the ACR/NEMA standards), several revisions have been developed for medical use. In 1993, the actual term DICOM (Digital Imaging and Communication in Medicine) was coined to describe the original standards and since that time additional revisions have occurred and continue to be considered to improve imaging compatibility with electronic records (termed: IHE Integrating the Healthcare Enterprise) and other digital systems. Newer revisions of the DICOM format incorporate the release year such as 'the 2007 version of DICOM' [6].

Since 1996, dentistry has been actively involved in the formulation of DICOM standards. This is when the American Dental Association joined the DICOM Committee. As a result, the DICOM v.3 includes definitions for image objects (also called 'data' ...for example: DX = diagnosis) with special categorization for intraoral projections (IO) as well as color photography. Participation by the ADA has continued with additional contributions including specifications related to different aspects of dental practice. Now most companies producing imaging devices (termed acquisition modalities) include the DICOM image identification system in the software that comes with the devices. There are also numerous other software products (termed Picture Archiving and Communication Systems - PACS) that have been further developed to allow for the comprehensive storage and retrieval, of digital DICOM images [7].

One important feature of the DICOM file is that it is fully encrypted at 128 bits. This allows HIPPA compliant electronic communication over the WEB. Having a standardized format also allows for viewing of images and associated data regardless of the proprietary acquisition modality or image display software that was used to acquire and display the imaging study. This feature allows cross-vendor connectivity or what is termed: 'Interoperability'. It also provides a means for dentists to communicate with their medical colleagues and each other as long as both providers use DICOM and HIPPA compliant WEB connections.

In Dentistry, there is also need for communication of non-radiologic information, for example $\mathrm{CAD} / \mathrm{CAM}$ digital data shared between a dentist and a laboratory fabricating a dental restoration. DICOM standards for this process were elucidated in 2010 by a DICOM WG-22 group representing dentistry. Several issues were considered during this meeting including the use of imaging in diagnosis, treatment simulation, treatment guidance, and tissue restoration using $\mathrm{CAD} / \mathrm{CAM}$ instrumentation. In addition the committee discussed how, with DICOM, images would be ordered, acquired from the acquisition modality (the device acquiring the information), processed, stored, and then communicated and displayed for reporting.

Allan G. Farman co-chaired the DICOM committee with their efforts detailed in a published article [8]. The specific work items included support for additional WG24 initiatives on DICOM for implants and the introduction of a DICOM supplement to support the full CAD/CAM prosthetic chain and DICOM surgical workflow issues in virtual and solid anatomic model fabrication-including a refinement of the 'Structured Display' and development of a 'Dental QueryRetrieve' application. In addition, the group sought to provide support for the joint WTG24/WG22 initiative on optical surface scanning.
Future work by the committee will include the development of guidelines for standardization of digital photographic structured displays for both intra as well as extraoral projections, the creation of templates for reports, the development of guidelines for presentation states including overlays used in dentistry, and surgical workflow issues within DICOM used in dental implantology [9].

Much work still needs to be done before dentistry is fully integrated with respect to DICOM and, in addition, the standardization of viewers used to retrieve information [6], bridging with newer acquisition devices, and the development of workflow issues associated with procedures such as implants. Additional work also relates to establishing connectivity with the many electronic Dental Records (EDR) software products currently available.

\section{Conformance Statement}

A dental digital x-ray machine, in DICOM terminology, is called an acquisition modality. Each acquisition modality includes a 'conformance statement'. Information contained in the Conformance Statement details how the x-ray system should be set up to allow communication between different products such as the viewing monitor or the digital record system and the acquisition device. It contains precise information that an IT or networking expert uses to provide a connection between various products provided by different vendors. This includes "information about the implementation model (provided services, data flow, functional definitions), the application entity specification (number of connections, transfer syntax, supported formats, etc.), and information about vendor specific (private) elements" [10].

As noted, the DICOM conformance statement contains the information necessary for IT to use when they connect different modalities. Nonetheless, as is stated by at least one modality provider, "the information contained in a DICOM conformance statement is not sufficient to ensure independent implementations will, in fact, be able to inter-operate" [11]. DICOM performance statements for various acquisition and other connected machines are typically available for download on the WEB. They can also be requested from the company providing the imaging product. The conformance statements for acquisition devices are standardized to allow comparison of DICOM devices and the ability of one product to communicate with another.

Simply put, the DICOM Conformance Statement is a 'diagram' of sorts that allows imaging equipment and management software to speak to each other. A dental x-ray taken by a specific intra-oral digital imaging machine can be downloaded to a server supplied by a different vendor and then displayed properly on a computer monitor made by another manufacturer. As is stated by the radiology society of North America's (RSNA) nontechnical introduction to DICOM Conformance "The Conformance Statement must describe how an activity handles associations (i.e. whether the activity initiates associations and accepts multiple associations) for each activity in the model. Some devices, such as the archives in a picture archiving and communication system (a PACS), must support multiple associations if performance is to be acceptable; otherwise, only a single activity (e.g. DICOM storage) can be handled at any given time" [12]. 


\section{Acquisition Devices}

As previous noted, an acquisition device is any instrument or machine that produces a digitized image. This includes CT, CBCT, MRI machines as well as ultrasound, and digital projection radiography. In dentistry, this also includes $\mathrm{x}$-ray machines that allow periapical, panoramic or cephalometric imaging, 3D computerized tomography, digital photography, and digitally-driven $\mathrm{CAD} / \mathrm{CAM}$ systems. In large part the newer standard dental digital imaging devices (e.g. intra-oral digital x-ray systems, panoramic imaging, and $\mathrm{CBCT}$ ) are DICOM compliant but, as noted previously, standards for DICOM compliance for some devices including $3 \mathrm{D}$ computerized tomography and $\mathrm{CAD} / \mathrm{CAM}$ systems and their interoperability with respect to some picture archiving and communications systems (PACS) have not been fully established.

\section{PACS (Picture Archiving and Communications Systems)}

A discussion of DICOM would not be complete without a brief discussion of picture archiving and communication systems (PACS). Most medical centers and large physician based HMOs in the US utilize PACS to manage digital DICOM files [13, 14] (Fig. 2). PACS software acts to integrate image acquisition, storage, retrieval, and viewing. In dentistry the use of PACS is primarily limited to academic centers and dental clinics in large hospital facilities where there is need for transmission of data between departments such as Radiology with Pathology, Oral Surgery, Oral Medicine, Periodontics, and Restorative Dentistry [15]. Or where there is communication between dentists and their physician colleagues. In the hospital setting DICOM compliant imaging is critical for a number of reasons, including HIPPA compliance. Image security is critical when the information is transmitted via public networks or the WEB. The 128 bit encryption offered by the DICOM format is largely effective in preventing hacking and theft of information [16].

\section{In-Office Servers and the Cloud}

Dentists that purchase acquisition devices typically install the software that comes with the machine(s) on the office server (their office hard drive). And the retrieval and display of images is usually fast as the file sizes of dental $\mathrm{x}$ ray surveys are small in comparison to MRIs or CT scans, which have much bigger files and are typically at the center of medical diagnosis. However, in the Dental office that takes CBCT and other complex imaging studies that have large file sizes, transmission and quick viewing can be problematic; this is because the more information that is stored on a hard drive (server) the slower the speed of retrieval. Speed may also be a problem if the data is intended to be transmitted between dental clinics at different locations.

The issue of speed can be mitigated by use of cloudbased systems that have been developed specifically to facilitate delivery of images within HIPPA compliance standards [17]. What the 'Cloud' offers is said to be a 'streamlined process for processing and sharing diagnostic images" [18].

The other issue related to PACS and DICOM image storage/retrieval systems is cost. Only large hospital based dental offices or large multi-clinic offices can usually afford to purchase a PACS and associated support (IT support) [19]. But 'Cloud' based systems cost considerably less as they are SaaS-based (i.e. they offer the product or 'software' as a service and customers pay only for what they use and as they use it) [20]. Maintenance of a purchased PACS system is

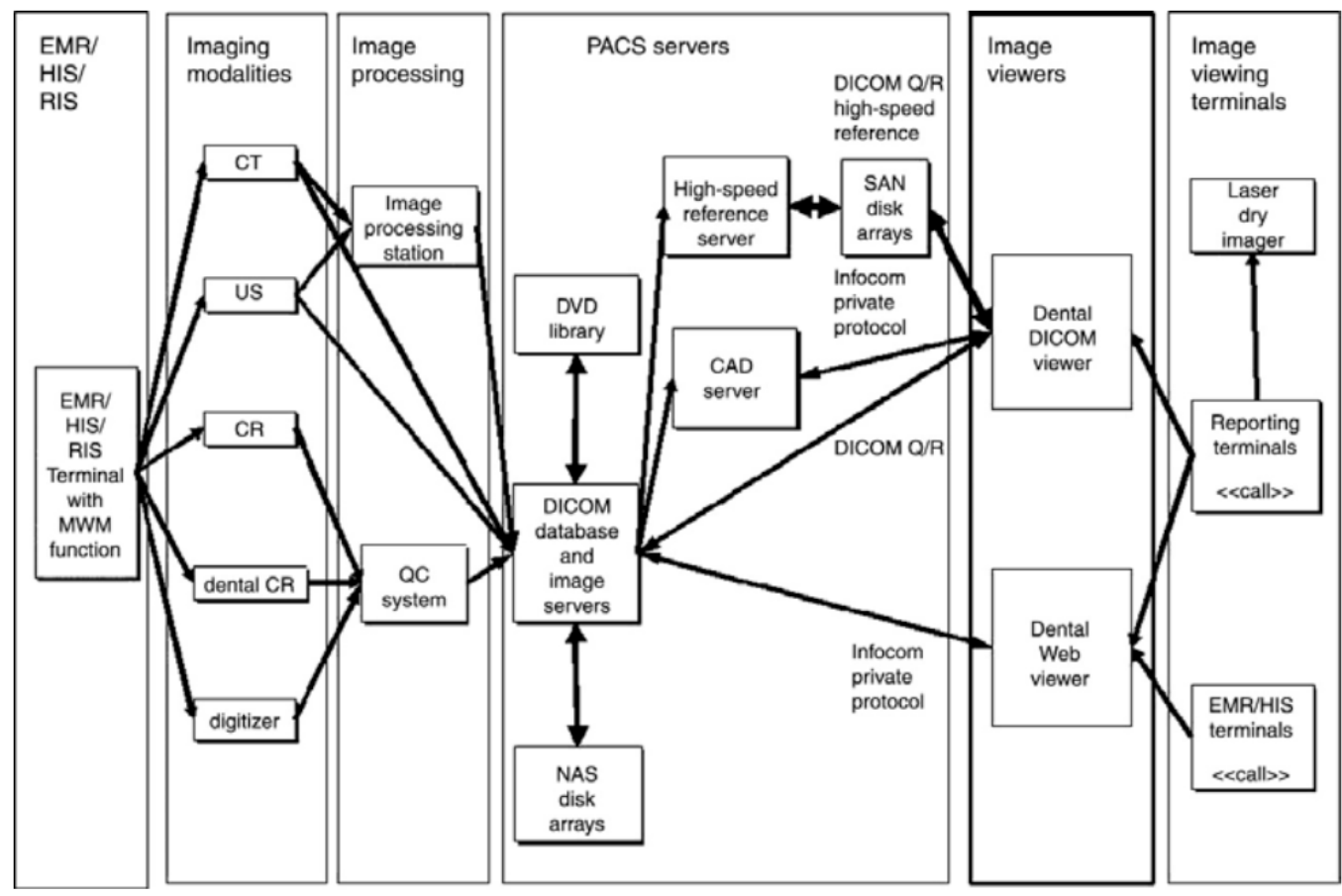

Fig. (2). Diagram showing the components of a hospital DICOM PACS: EMR (electronic medical records) communicates with the image acquiring/display functions (courtesy of EBM Technologies). 
also costly so 'Cloud' approaches to DICOM document storage may be much more affordable, especially to the smaller or medium sized dental clinic that needs to store many large sized files.

\section{TERMINOLOGY}

To fully understand 'DICOM' it is important to know what the various terms associated with the term mean. The following are just a few of the terms and meanings that are incorporated into 'DICOM'. Although these terms are not now typically used by dentists in their day to day professional communication with IT personnel, if digital imaging is being considered or has been implemented and DICOM compliance becomes the standard for communication in dentistry they are likely to become commonplace.

1) Association: the communication connection that is established between two DICOM applications by which DICOM information is exchanged. One or more associations may be supported simultaneously.

2) Attributes: these are items that describe something and within DICOM, attributes are used to describe information objects.

3) Composite objects: the objects that are defined in DICOM corresponding to multiple or parts of multiple entities in the 'entity-relationship' (E-R) model.

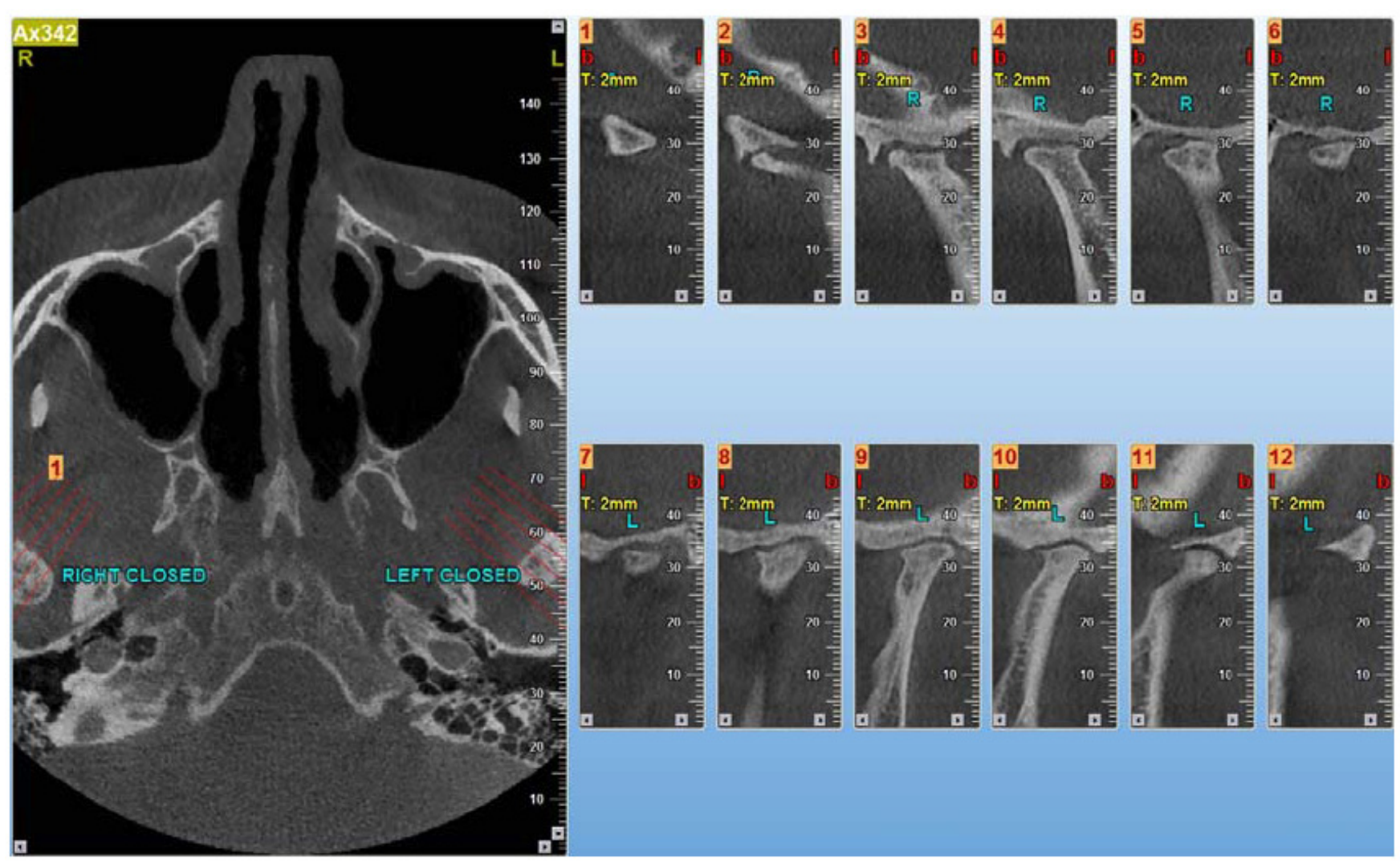

4) Data elements: a description of the contents of data sets or descriptive attributes that 'provide the characteristics of entities' in the E-R model.

5) Data set: In the E-R model this is the formal description of entities such as patients, equipment, images, etc. and how they are related based on an informationorganization perspective.

6) Unique identifier: This is a numeric construct that is used when an entity is referenced. It is a unique 'name' that allows the finding and retrieval of the desired entity and allows its distinction from other entities.

7) Value representation (VR): Within DICOM, it is the description of how the attribute value is represented, for example as text, binary data, or patient name.

8) Library: this refers to the generally accepted terms describing attributes such as patient name, identification number, date of birth, etc.

\section{DICOM Use in Dentistry}

Digital systems and DICOM imaging standards are finding application in the fields of orthodontics, oral surgery, oral medicine, and implants and are being utilized in hospital and dental school settings [2, 12, 19, 21-24] (Fig. 3).

\section{Orthodontics}

Imaging of the craniofacial region is strategic to treatment planning in orthodontics. Historically, orthodontic pa-

Fig. (3). This figure shows a CBCT DICOM imaging study and demonstrates the slice pattern (left) and slices (right side) taken through the bilateral TMJ region. A DICOM image allows for the inclusion of data not otherwise possible with legacy film. 
tient evaluation has included the use of 2-D static imaging techniques to assess three-dimensional craniofacial structures. A relatively recent innovation in diagnostic imaging is cone beam computed tomography (CBCT) which provides $3-\mathrm{D}$ views of the craniofacial area. It has been suggested that this new 3-D imaging technology may improve diagnosis, treatment options, final treatment results, outcome measure assessment, and the monitoring of the patient over time [25].

Grauer, et al. [24], discuss DICOM as it relates to CBCT imaging in orthodontics. This includes measurement issues, creation of 2-D radiographs from DICOM CBCT files, segmentation engines and multimodal images, the registration and superimposition of 3-D images, and applications for quantitative analysis and 3-D surgical prediction.

Converting CBCT images into DICOM is the first step in converting two dimensional images into 3-D files. Sophisticated software programs that are available include 3dMDvultus software (3dMD, Atlanta, Ga), Dolphin Imaging (Dolphin Imaging, Chatsworth, California), and InVivoDental (Anatomage, San Jose, California). There is also freeware. These software programs are able to transform CBCT DICOM images along different planes to allow the visualization of specific regions of interest. Angles can be changed and scaled to improve visualization and multiple threshold filters allow the viewer to differentiate tissue density and apply clipping tools and transparency filters for viewing soft and hard tissues.

However, quantitative assessment in orthodontic treatment planning using CBCT 3-D converted to 2-D rendering has limitations. For example, the visual landmarks identified in 2-D may be difficult to visualize or locate on a curved 3-D surface. In addition, factors such as contrast, movement during acquisition, the presence of metal, signal-to-noise ratio, and threshold filters applied by an operator may impact a rendered image. Anatomic landmarks need to be localized within a stack of slices and as a result, the accuracy and reliability of measurements on CBCT 3-D images can vary depending on which slice is utilized. However, it is concluded [24] that it is still more accurate to locate landmarks within stacked slices that attempting to locate them in a rendered 3$\mathrm{D}$ volume. A caveat is that data included within a DICOM file should be interpreted with caution in providing orthodontic diagnosis because the available tools in the 3-D software systems have not been fully validated in terms of 'accuracy and precision'.

\section{Oral Surgery}

Clinical pre-surgical planning is an area where DICOM based files may be useful as DICOM CBCT images provide information that is useful in defining general anatomy and determining the extent of pathology. However, some evidence suggests that the complexity of the DICOM file data may be problematic when applied to research. A study exploring CBCT surveys and the properties of the images used to evaluate maxillofacial bone grafts indicates that the included DICOM files, while useful for assessing issues associated with graft viability, may also, because of their complexity, compromise comparison of data between studies
[26]. However, this is not likely to represent a significant problem in terms of clinical practice.

Surgical implant planning is another area where DICOM based files in CBCT can be helpful. As an example, it is reported in one article that data from a CT scan taken with an intraoral template in place was than stored on a CD-ROM in DICOM3 format and later uploaded to an implant software program, where it was used to calculate the parameters necessary for site preparation [22]. This strategy allows view of the implant placement in three dimensions. Using DICOM imaging in this manner may improve surgical accuracy. And where exactness is important, such as in cases where there is little space such as in the atrophic maxillae or where there has been a sinus lift, the use of 3-D CBCT DICOM files may be critical to therapeutic success. Computer assisted navigation systems are in development to provide real time evaluation but, as noted by the above authors, DICOM connectivity issues have yet to be resolved [22].

\section{Oral Medicine}

With CBCT DICOM files not only can pathology be readily identified, it can be measured in three dimensions and assessed in terms of relative volume and evaluated for relative bone density. In addition, CBCT DICOM imaging studies may help in the assessment and management of sleep apnea, snoring, and airway obstruction as the shape and contours of upper airway passages can be analyzed in three dimensions. All of the DICOM orthodontic software programs mentioned above allow measurement of airway volume, thus providing potential for a 'virtual endoscopy' [24].

\section{DICOM Concerns}

One concern related to the use of DICOM is in relation to the diagnosis of caries. Specifically, the question is whether caries can be reliably diagnosed when images are viewed on monitors with different specifications? Limited research suggests that monitor type may not make a difference with respect to the identification of caries, regardless of whether the digital image is presented on a DICOM pre-calibrated monitor or a monochromatic monitor [1].

Another issue relates to the digital compression of images. File compression associated with DICOM storage could alter a viewed image in such a way as to hinder diagnosis. However, studies involving assessment of root fracture suggests that it does not, as images evaluated in at least two studies failed to show significant differences between uncompressed and compressed images in the detection of root fractures $[27,28]$. Other studies evaluating compression issues also indicate that compression generally does not affect landmark identification in lateral cephalometric digital radiographs [29] or with respect to reproducible cephalometric points [30].

\section{Innovations in DICOM}

Two of the more interesting innovations with DICOM based imaging include internet image transfer and the use of mobile devices as viewers [31-33]. DICOM images can be transferred via the internet because they are fully encrypted. This allows the communication of information between pro- 
viders in nearly real time. It also may reduce the possibility of error introduction associated with compact disc transfer [34].

Thus, a dentist that needs an oral surgical or oral medicine consultation related to suspect pathology could send a DICOM based imaging survey via the internet and get immediate feedback while the patient is still in the treatment room. And the transferred information would be HIPPA compliant. This represents a significant step forward in the communication of digital files. And recent advances in digital display associated with mobile devices such as tablets and phones adds another layer to the internet communication possibilities of DICOM based images.

\section{CONCLUSION}

There is little information documenting the number of dentists or dental clinics that use DICOM in dentistry or PACS or Cloud storage in the US although published studies suggest that this standard and these retrieval/storage systems associated with its use are increasing $[2,15]$. However with the full implementation of digital standards (electronic health records) associated with national health care in the US and other countries, the sharing of images between offices in multi-site dental practices, and the continuing pressure for dental compliance with national and international imaging standards as well as the convenience that the technology affords in terms of web transmission, it is likely that moderate to large dental facilities will begin to move towards storage implementation using DICOM and 'Cloud' based storage and retrieval; and this may allow smaller group practices to follow their lead $[2,15,35]$.

\section{CONFLICT OF INTEREST}

The author confirms that this article content has no conflict of interest.

\section{ACKNOWLEDGEMENTS}

Declared none.

\section{REFERENCES}

[1] Hellén-Halme K, Nilsson M, Petersson A. Effect of monitors on approximal caries detection in digital radiographs: standard versus precalibrated DICOM part 14 displays: an in vitro study. Oral Surg Oral Med Oral Pathol Oral Radiol Endod 2009; 107: 716-20.

[2] Draenert FG, Erbe C, Zenglein V, et al. 3D analysis of condylar position after sagittal split osteotomy of the mandible in mono- and bimaxillary orthognathic surgery - a methodology study in 18 patients. J Orofac Orthop 2010; 71: 421-9.

[3] Available from: http://dicom.offis.de/dcmintro.php.en. [Accssed $1 / 7 / 15]$.

[4] Dale M. DICOM in Dentistry: Interoperability and X-rays. [Accssed] 1/8/15. Available from: http://www. learndigital.net/articles/2004/dicon_dentistry.htm

[5] Available from: http://www.dicomanalyser.co.uk/ html/ introduction.htm. [Accssed 1/7/15].

[6] Farman AG. Applying DICOM to dentistry. J Digit Imaging 2005; 18: 23.

[7] http://www.ada.org/en/science-research/dental-standards/standardscommittee-on-dental-informatics/standards-technical-specificationsand-technical-reports. [Accssed 1/7/15].
[8] Available from: http://dental.healthimaginghub.com/portals/dentalpacs-news-article/2219-dicom-the-future-of-x-ray-imaging-indentistry. [Accssed 1/7/15].

[9] Farman AG, Lapp RP. Image file interoperability for data protection, communication and trans-system connectivity. Orthod Craniofac Res 2003; 6 (1): 151-5.

[10] Available from: http://www.healthcare.philips.com/main/about/ connectivity/dicom_conformance_main.wpd; Assessed 1/4/15.

[11] Available from: http://medxchange.com/support/dicom-conformancestatement/. [Accssed 1/5/15].

[12] Indrajit IK. Digital imaging and communications in medicine: A basic review. Indian J Radiol Imaging 2007; 17: 5-7

[13] http://link.springer.com/chapter/10.1007\%2F978-3-642-243523_27. [Accssed 1/8/15].

[14] Gale ME, Gale DR. DICOM modality worklist: an essential component in a PACS environment. J Digit Imaging 2000; 13: 101-8.

[15] Chen SK. Integration of the digital imaging and communications in medicine standard into an oral and maxillofacial image management and communication system. Oral Surg Oral Med Oral Pathol Oral Radiol Endod 2001; 91: 235-8

[16] De Backer AI, Mortelé KJ, De Keulenaer BL. Picture archiving and communication system--Part one: Filmless radiology and distance radiology. JBR-BTR 2004; 87: 234-41.

[17] http://venturebeat.com/2014/07/17/dicom-grid-raises-6m-fordigital-medical-imaging-platform/. Accssed 1/5/15.

[18] http://info.radiologyservicesonline.com/blog-0/bid/137782/ Understanding-DICOM-and-the-Cloud. [Accssed 1/7/15].

[19] Ohtsuka M, Nakamoto T, Konishi M, et al. Experience of using a filmless system in dental practice at Hiroshima University Hospital, a former university dental hospital. [Article in Japanese] Nihon Hoshasen Gijutsu Gakkai Zasshi 2011; 67: 673-8.

[20] http://info.radiologyservicesonline.com/blog-0/bid/137782/ Understanding-DICOM-and-the-Cloud. [Accssed 1/7/15].

[21] Mupparapu M, Binder RE, Cummins JM, et al. Implementation of a Digital Radiographic Image Acquisition and Retrieval System (DRIARS) using a wireless network in an orthodontic department. J Contemp Dent Pract 2008; 9: 115-23.

[22] Rubio Serrano M, Albalat Estela S, Peñarrocha Diago M, et al. Software applied to oral implantology: update. Med Oral Patol Oral Cir Bucal 2008; 13: E661-5.

[23] Nair MK, Pettigrew JC Jr, Loomis JS, et al. Enterprise-wide implementation of digital radiography in oral and maxillofacial imaging: the University of Florida Dentistry System. J Digit Imaging 2009; 22: 232-41.

[24] Grauer D, Cevidanes LS, Proffit WR. Working with DICOM craniofacial images. Am J Orthod Dentofacial Orthop 2009; 136: 46070.

[25] Jyothikiran H, Shanthara JR, Subbiah P, et al. Craniofacial imaging in orthodontics--past present and future. Int J Orthod (Milwaukee) 2014; 25: 21-6.

[26] Spin-Neto R, Marcantonio E Jr, Gotfredsen E, et al. Exploring CBCT-based DICOM files. A systematic review on the properties of images used to evaluate maxillofacial bone grafts. J Digit Imaging 2011; 6: 959-66.

[27] Noujeim M, Geha H, Shintaku W, Bechara B, Kashi KA. Effect of JPEG compression on the diagnostic accuracy of periapical images in the detection of root fracture. Dent Traumatol 2012; 28(3): 2337.

[28] Melo SL, Haiter-Neto F, Correa LR, et al. Comparative diagnostic yield of cone beam CT reconstruction using various software programs on the detection of vertical root fractures. Dentomaxillofac Radiol 2013; 42: 20120459.

[29] Abdelkarim A, Nummikoski P, Gakunga P, et al. Effect of JPEG2000 compression on landmark identification of lateral cephalometric digital radiographs. Am J Orthod Dentofacial Orthop 2010; 138: 518-24. 
[30] Duarte H, Vieck R, Siqueira DF, et al. Effect of image compression of digital lateral cephalograms on the reproducibility of cephalometric points. Dentomaxillofac Radiol 2009; 38: 393-400.

[31] Zhang J, Sun J, Stahl JN. PACS and Web-based image distribution and display. Comput Med Imaging Graph 2003; 27: 197-206.

[32] Raman B, Raman R, Raman L, et al. Radiology on handheld devices: image display, manipulation, and PACS integration issues. Radiographics 2004; 24: 299-310.
[33] Andrade R, Wangenheim A, Bortoluzzi MK. Wireless and PDA: a novel strategy to access DICOM-compliant medical data on mobile devices. Int J Med Inform 2003; 71: 157-63.

[34] McEvoy FJ, Svalastoga E. Security of patient and study data associated with DICOM images when transferred using compact disc media. J Digit Imaging 2009; 22: 65-70.

[35] Cardelli P. New online management software and DICOM viewer for dentistry. Int J Comput Dent 2011; 14:147-53.

Received: October 07, 2014

Revised: January 17, 2015

Accepted: May 25, 2015

(C) Jeff Burgess; Licensee Bentham Open.

This is an open access article licensed under the terms of the Creative Commons Attribution Non-Commercial License (http://creativecommons.org/licenses/by-nc/3.0/) which permits unrestricted, non-commercial use, distribution and reproduction in any medium, provided the work is properly cited. 